\title{
Pancreatic cancer: let us focus on cachexia, not just sarcopenia!
}

\author{
Savio George Barreto*,1,2 \\ ${ }^{1}$ Division of Surgery \& Perioperative Medicine Flinders Medical Centre, Bedford Park, Adelaide, South Australia, Australia \\ ${ }^{2}$ College of Medicine \& Public Health, Flinders University, South Australia, Australia \\ *Author for correspondence: Tel.: +61 8 82045511; georgebarreto@yahoo.com
}

\begin{abstract}
"'Rather than focusing our efforts on sarcopenia (a mere component of cachexia), we need to concentrate on the main problem itself, which is cancer cachexia. Diagnosing cachexia in the patient and instituting appropriate management from the outset appears to be the way forward."
\end{abstract}

First draft submitted: 15 May 2018; Accepted for publication: 31 July 2018; Published online: 7 September 2018

Keywords: morbidity • mortality • outcomes • survival

In most circumstances, though not uniformly, advanced or metastatic cancer is clinically represented by the appearance of a cachectic and moribund patient. Cancer cachexia is associated with treatment failure [1] and poor overall survival $[2,3]$ even in patients with treatable disease. Pancreatic cancer is no exception. While more than $75 \%$ of patients with pancreatic cancer present when the disease is no longer amenable to a curative resection [4], cachexia signals poor outcomes even for the remaining $25 \%$ who are offered a surgical resection with an intent to cure [5-7]. Whether this is a representation of pancreatic cancer being a systemic disease from its onset remains a matter of conjecture.

In recent years, there has been a dramatic increase in the number of publications aiming to correlate radiologically detectable sarcopenia and outcomes in cancer. This is an offshoot of the understanding that sarcopenia, an involuntary loss of skeletal muscle mass and/or strength, is a major component of cancer cachexia [8]. A PubMed search (up to the date 15 May 2018) carried out using the search terms 'sarcopenia', 'cancer' and 'survival' returned 404 studies with $331(82 \%)$ of these published within the last 5 years. In pancreatic cancer, too, there have been many such studies. However, to date, there has been no conclusive evidence to support an association between sarcopenia and pancreatic cancer survival [7,9].

While considerable time and effort is being invested into such research, a couple of questions appear to surface, 'do we need to prove an association between sarcopenia and pancreatic cancer survival'? and 'to what end are we seeking to elucidate this association'? The reason for these questions stems from the fact that cancer cachexia is a disease continuum and making assumptions on a single cross-sectional imaging finding of sarcopenia does not provide us sufficient information to understand where in the cancer cachexia spectrum a particular patient lies. Should our efforts not be focused on improving patient outcomes, especially quality of life? To do so, we would need to subclassify a patient within the cachexia spectrum and focus on the drivers of cachexia in that patient. Sadly today, cancer cachexia is a neglected condition with no established treatment [10].

We know that cancer cachexia is a disease continuum characterized by an ongoing loss of skeletal muscle mass (with or without loss of fat mass) that cannot be fully reversed by conventional nutritional support eventually leading to progressive functional impairment [11]. There exists a complex interplay of reduced food intake and an abnormal metabolism that drive the effects of the tumor and systemic inflammation [11]. Fearon and colleagues [11] deconstructed the cachexia continuum into three stages; namely, precachexia, cachexia and refractory cachexia with an aim to provide a framework for future interventional research. While refractory cachexia signifies a preterminal stage of very advanced or a rapidly progressive cancer wherein active intervention to reverse weight loss may be less effective and emphasis should be on symptom control, the other two stages offer the prospect of active intervention. Precachexia, characterized by anorexia and impaired glucose tolerance accompanied by weight loss $\leq 5 \%$ of body 
weight, presents an opportunity to monitor these patients and institute preventive intervention strategies. As for cachexia, the possibility for multimodal management focusing on reversible contributory factors seems attractive.

In pancreatic and periampullary cancer, there has been some interesting work combining radiology (including body composition analysis), molecular biology and metabolomics with a clinical correlation (survival) [9]. The study by Stretch and colleagues [9] supports the lack of an association between sarcopenia and survival in pancreatic cancer but more importantly presents novel evidence for the effect of myosteatosis on cancer survival in patients amenable to surgery - a reflection of the underlying ill health in these patients. The driver of myosteatosis in pancreatic cancer remains to be clarified. However, cancer cachexia, with its well-known effects on muscle wasting secondary to decreased protein synthesis, impaired regeneration and increased degradation [12,13], is a certain front-runner. Additionally, muscle depletion in patients on palliative chemotherapy for pancreatic [14] and ampullary [15] cancers has been shown to be a poor prognostic factor for survival.

Recent preclinical experimental work by Nissinen and colleagues [16] blocking ACVR2 ligands, the mediators of the negative effects of myostatin and activin on muscle mass, has shown some promising results although more work is needed to understand the mechanisms involved.

Another molecule that has been studied in cancer cachexia is the orexigenic hormone, ghrelin. Ghrelin transmits hunger signals from the periphery to the CNS [17] resulting in an increased appetite. It also stimulates growth hormone release, prevents muscle catabolism and promotes gut motility thereby contributing to an increase in bodyweight [18]. Although the current evidence on ghrelin may not be robust enough to support its use in the treatment of cancer cachexia [18], further research into its benefits is certainly warranted.

Pancreatic exocrine insufficiency is an often forgotten contributor to malnutrition in pancreatic cancer. Pancreatic exocrine insufficiency may be seen in patients with resectable cancers after surgery, or in advanced cancer patients - an effect of the tumor, itself [19]. Pancreatic enzyme replacement therapy has been shown to improve weight gain [20], as well as, survival in patients with unresectable disease [21].

Over the years, there has been a growing awareness of the importance of adopting a multipronged interdisciplinary approach to the treatment of cancer cachexia [22]. Such an approach would include targeting reversible causes of reduced food intake [23], using omega 3 fatty acid supplements [24], as well as encouraging physical exercise [25] alongside standard treatments; namely, surgery, chemotherapy and radiation therapy. Solheim and colleagues [26] have completed a Phase II trial demonstrating the safety and feasibility of a multimodal intervention comprising n-3 polyunsaturated fatty acid, nutritional supplements, exercise and celecoxib in patients with incurable pancreatic cancer undergoing palliative chemotherapy. They have now embarked on a Phase III trial, with the acronym MENAC, which stands for Multimodal Intervention - Exercise, Nutrition and Anti-inflammatory medication in Cachexia (NCT02330926) [10]. This prospective, multicenter, randomized controlled trial will recruit patients from multiple sites in Europe and Canada with an aim to attenuate or prevent weight and muscle loss and improve physical function in patients with lung or pancreatic cancer, receiving anticancer therapy. The results of this trial are eagerly awaited. The addition of pancreatic enzyme replacement therapy to such multimodality treatments of cachexia in pancreatic cancer merits consideration.

In conclusion, cachexia in pancreatic cancer is likely a phenomenon that exists at the time of diagnosis, irrespective of the stage of the disease. Rather than focusing our efforts on sarcopenia (a mere component of cachexia), we need to concentrate on the main problem itself, which is cancer cachexia. Diagnosing cachexia in the patient and instituting appropriate management from the outset appears to be the way forward. There are sufficient opportunities for meaningful research in this vast, largely underappreciated clinical entity that contributes significantly to the morbidity and mortality in patients with pancreatic cancer. Obtaining this information would help us institute appropriate, supportive therapies for our patients with the overarching aim of improving quality of life, and maybe even overall survival.

Financial \& competing interests disclosure

The author has no relevant affiliations or financial involvement with any organization or entity with a financial interest in or financial conflict with the subject matter or materials discussed in the manuscript. This includes employment, consultancies, honoraria, stock ownership or options, expert testimony, grants or patents received or pending, or royalties.

No writing assistance was utilized in the production of this manuscript. 


\section{References}

Papers of special note have been highlighted as: $\bullet$ of interest; $\bullet \bullet$ of considerable interest

1. Mauri D, Tsiara A, Valachis A et al. Cancer cachexia: global awareness and guideline implementation on the web. BMJ Support. Palliat. Care 3(2), 155-160 (2013).

2. Dewys WD, Begg C, Lavin PT et al. Prognostic effect of weight loss prior to chemotherapy in cancer patients. Eastern Cooperative Oncology Group. Am. J. Med. 69(4), 491-497 (1980).

3. Fearon KC, Voss AC, Hustead DS. Definition of cancer cachexia: effect of weight loss, reduced food intake, and systemic inflammation on functional status and prognosis. Am. J. Clin. Nutr. 83(6), 1345-1350 (2006).

4. Barreto S, Windsor J. Justifying vein resection with pancreatoduodenectomy. Lancet Oncol. 17(3), e118-e124 (2016).

5. Bachmann J, Heiligensetzer M, Krakowski-Roosen H, Buchler MW, Friess H, Martignoni ME. Cachexia worsens prognosis in patients with resectable pancreatic cancer. J. Gastrointest. Surg. 12(7), 1193-1201 (2008).

6. Pausch T, Hartwig W, Hinz U et al. Cachexia but not obesity worsens the postoperative outcome after pancreatoduodenectomy in pancreatic cancer. Surgery 152 (3 Suppl. 1), S81-S88 (2012).

7. Ozola Zalite I, Zykus R, Francisco Gonzalez M et al. Influence of cachexia and sarcopenia on survival in pancreatic ductal adenocarcinoma: a systematic review. Pancreatology 15(1), 19-24 (2015).

- Most updated systematic review of sarcopenia in pancreatic cancer.

8. Baracos VE, Reiman T, Mourtzakis M, Gioulbasanis I, Antoun S. Body composition in patients with non-small-cell lung cancer: a contemporary view of cancer cachexia with the use of computed tomography image analysis. Am. J. Clin. Nutr. 91(4), 1133S-1137S (2010).

9. Stretch C, Aubin JM, Mickiewicz B et al. Sarcopenia and myosteatosis are accompanied by distinct biological profiles in patients with pancreatic and periampullary adenocarcinomas. PLoS ONE 13(5), e0196235 (2018).

10. Solheim TS, Laird BJA, Balstad TR et al. Cancer cachexia: rationale for the MENAC (Multimodal-Exercise, Nutrition and Anti-inflammatory medication for Cachexia) trial. BMJ Support. Palliat. Care 8, 258-265 (2018).

- Manuscript presents the rationale for the trial looking at a multipronged approach to cancer cahcexia.

11. Fearon K, Strasser F, Anker SD et al. Definition and classification of cancer cachexia: an international consensus. Lancet Oncol. 12(5), 489-495 (2011).

-. Manuscript presents a thorough systematic review of the evidence on cachexia and provides definitions and a simplified understanding of the disease entity to facilitate the development of future studies.

12. Smith KL, Tisdale MJ. Increased protein degradation and decreased protein synthesis in skeletal muscle during cancer cachexia. $B r$. J. Cancer 67(4), 680-685 (1993).

13. Talbert EE, Guttridge DC. Impaired regeneration: a role for the muscle microenvironment in cancer cachexia. Semin. Cell Dev. Biol. 54, 82-91 (2016)

14. Choi Y, Oh DY, Kim TY et al. Skeletal muscle depletion predicts the prognosis of patients with advanced pancreatic cancer undergoing palliative chemotherapy, independent of body mass index. PLoS ONE 10(10), e0139749 (2015).

15. Cho KM, Park H, Oh DY et al. Skeletal muscle depletion predicts survival of patients with advanced biliary tract cancer undergoing palliative chemotherapy. Oncotarget 8(45), 79441-79452 (2017).

16. Nissinen TA, Hentila J, Penna F et al. Treating cachexia using soluble ACVR2B improves survival, alters mTOR localization, and attenuates liver and spleen responses. J. Cachexia Sarcopenia Muscle 9(3), 514-529 (2018).

17. Kojima M, Kangawa K. Structure and function of ghrelin. Results Probl. Cell Differ. 46, 89-115 (2008).

18. Khatib MN, Shankar AH, Kirubakaran R et al. Ghrelin for the management of cachexia associated with cancer. Cochrane Database Syst. Rev. 2, CD012229 (2018)

19. Toouli J, Biankin AV, Oliver MR, Pearce CB, Wilson JS, Wray NH. Management of pancreatic exocrine insufficiency: Australasian Pancreatic Club recommendations. Med. J. Aust. 193(8), 461-467 (2010).

20. Bruno MJ, Haverkort EB, Tijssen GP, Tytgat GN, van Leeuwen DJ. Placebo controlled trial of enteric coated pancreatin microsphere treatment in patients with unresectable cancer of the pancreatic head region. Gut 42(1), 92-96 (1998).

21. Dominguez-Munoz JE, Nieto-Garcia L, Lopez-Diaz J, Larino-Noia J, Abdulkader I, Iglesias-Garcia J. Impact of the treatment of pancreatic exocrine insufficiency on survival of patients with unresectable pancreatic cancer: a retrospective analysis. BMC Cancer 18(1), 534 (2018)

22. Granda-Cameron C, DeMille D, Lynch MP et al. An interdisciplinary approach to manage cancer cachexia. Clin. J. Oncol. Nurs. 14(1), 72-80 (2010)

23. Del Fabbro E, Hui D, Dalal S, Dev R, Nooruddin ZI, Bruera E. Clinical outcomes and contributors to weight loss in a cancer cachexia clinic. J. Palliat. Med. 14(9), 1004-1008 (2011).

24. de Aguiar Pastore Silva J, Emilia de Souza Fabre M, Waitzberg DL. Omega-3 supplements for patients in chemotherapy and/or radiotherapy: a systematic review. Clin. Nutr. 34(3), 359-366 (2015). 
25. Speck RM, Courneya KS, Masse LC, Duval S, Schmitz KH. An update of controlled physical activity trials in cancer survivors: a systematic review and meta-analysis. J. Cancer Surviv. 4(2), 87-100 (2010).

26. Solheim TS, Laird BJA, Balstad TR et al. A randomized Phase II feasibility trial of a multimodal intervention for the management of cachexia in lung and pancreatic cancer. J. Cachexia Sarcopenia Muscle 8(5), 778-788 (2017). 